\title{
On Reliability in Evaluating the Awareness of Health Hazard of Tobacco Smoking of a Group Bangladeshi Students
}

\author{
KC Bhuyan ${ }^{1 *}$ and Urmi AF $^{2}$ \\ Professor [Retired] of Statistics, Jahangirnagar University, Bangladesh \\ Department of Mathematics, American International University, Bangladesh
}

*Corresponding author: KC Bhuyan, Professor [Retired] of Statistics, Jahangirnagar

University, Dhaka, Bangladesh.

\begin{abstract}
The present analysis was based on data related to knowledge about health hazard of tobacco smoking of a group of students. The data were collected from 798 students of American International University-Bangladesh during the academic session 2012-2013. Around 87\% of the investigated students were high in awareness of health hazard of tobacco smoking but still $32.3 \%$ students were smokers. There was none who was low in awareness. The awareness had been measured by 20 items. Though most of the students were highly aware of the problem still a good number of them were smokers. Thus, a question, naturally, arose whether measure of awareness was reliable. In this paper, reliability measure had been done by Cronbach's alpha [1]. The value of $\alpha$ is 0.94 indicating that the evaluation of knowledge of health hazard of tobacco smoking was excellent. Reliability had also been measured by Spearman-Brown formula and the value of the coefficient was 0.92 .
\end{abstract}

Keywords: Awareness of health hazard of tobacco smoking; Smokers; Reliability of measure of awareness; Spearman-brown formula for reliability; Cronbach's alpha

\section{Introduction}

Reliability in statistics and psychometrics is the overall consistency of a measure where measure is done by different rater. It is related to test and deals with consistency, precision, repeatability, trustworthiness [2]. A measure is said to have a high reliability if it produces similar results under consistent condition.

Consistency is of two types, viz. (a) absolute consistency which concerns with consistency of scores of individuals, (b) relative consistency which concerns with the consistency of the positions or ranks of individuals in the group $[3,4]$. This means that reliability is a property of scores of a measure when the measure is done using several items, qualitative or quantitative, especially in the field of education, psychology, health science and even in business.

The reliability is measured by a coefficient which provides the information about the dependability of the measurement by a number of ranges from 0 to 1 . The reliability coefficient is given by

$$
\mathrm{P}_{\mathrm{xx}}=\mathrm{o}_{\mathrm{T}}^{2} / \mathrm{o}_{\mathrm{x}}^{2}=1-\left({\sigma_{\epsilon}^{2}}^{2} / \mathrm{o}_{\mathrm{x}}^{2}\right) \text {, where, }
$$

$$
\mathrm{o}_{\mathrm{x}}^{2}={\boldsymbol{O}_{\mathrm{T}}}^{2}+\mathrm{o}_{\boldsymbol{\epsilon}}^{2} \text {, where, } \mathrm{X}=\mathrm{T}+\boldsymbol{\epsilon}
$$

Here, $\mathrm{T}=$ true score, $\boldsymbol{\epsilon}=$ measurement error, $\mathrm{X}$ is observed score; $\mathrm{X}^{\prime}$ is the measure of $\mathrm{X}$ when it is split or it is the measure by different raters.

There are different measures of reliability. One of the measures has been done by Spearman- Brown formula [5]. Other measures of reliability were suggested by many eminent statisticians, notable among them are Cochran, Cohen, Cronbach, Kudar and Richardson, Guttman, Michael, Olga, Maxwell Chakrabarty [6-14]. Chakrabarty suggested the split-half method of measuring coefficient of reliability using Spearman-Brown formula with a modified approach.

However, due to different proposed measure, reliability of a test may vary from one sample to another [15]. According to the variation in measure, a test is excellently reliable if its coefficient is $0.90^{+}$; Cronbach's [8] reliability is good if coefficient ranges from 0.80 to 0.90 . The test does not contribute if the coefficient becomes 0.50 or less. 
The objective of the present study was to evaluate how much reliable was the measure of awareness of youth regarding health hazard of tobacco smoking. Because, in a separate study, it was reported that youth who were more aware of the problem of health hazard of tobacco smoking, a good number of them was smokers. As was expected the more awareness of the problem would lead to less smokers in percentage [16]. Otherwise, the evaluation of awareness would not be reliable. The reliability measure would indicate whether youth are really aware of the problem. In a separate study among the students in abroad it was reported that a good number of students were aware of the problem but rate of smokers was not less [17]. So, naturally, a question was arisen whether measure of awareness was reliable. Though such study was not reported earlier, we made an attempt to study the reliability of the measure of awareness. Because, if the measure of awareness is reliable and still higher proportion of youth are smokers, alternative way needs to be suggested to discourage the youth so that they avoid smoking.

\section{Methodology}

The data was collected from 798 randomly selected students of American International University-Bangladesh during academic session summer 2012-13. During the session there were in total 11009 students. They were registered in sections of a course. In each section, the number of students is approximately 40 and classes of all faculties run at the same time.

It was decided to provide the pre-tested questionnaires to the students of a section of different faculties attending the classes at a time so that no student gets questionnaire twice or more.

The questionnaire was provided to the students covering $10 \%$ of each faculty and they were requested to return the filled in questionnaires to their respective teachers in the subsequent class/ classes. However, the filled in questionnaires were returned by 798 students. The analytical results of the data collected from 798 students had been reported in a separate paper and the present analysis was based on the evaluation of awareness of health hazard of tobacco smoking expressed in the paper [16].

There were 20 questions to evaluate awareness of health hazard. Each question had closed answers like 'True', 'False' and 'Don't Know'. The affirmative answer towards health hazard of tobacco smoking was assigned ' 3 ' followed by ' 2 ' with less awareness. The number ' 1 ' is assigned for negative answer towards awareness. The maximum score, as was expected, for high level of awareness was 60 and for low level of awareness the total score was 20. Khatun and Bhuyan had classified the students as highly aware (total score $40^{+}$), medium in awareness (total score 30-40) and low in awareness (total score <30). Among the sample students 697 were highly aware of the problem and 101 were medium in awareness. In other papers, it was reported that smoking habit and awareness were significantly associated indicating that lower proportion $(32.3 \%)$ of highly aware students were smokers $[18,19]$.

In spite of high level of awareness among majority (87.3\%) of the students, a good number of students were smokers. As higher proportion of respondents were aware, the much lower could be the proportion of smokers. But the incidence of smoking was not so lower compared to that in general [16]. Thus, a question, naturally, arose whether the evaluation of the awareness was reliable or not. In this paper, the reliability of measure of awareness of health hazard of smoking was studied by Cronbach LJ [1] alpha, where the Cronbach's alpha is given by

$$
\alpha=\frac{k}{k-1}\left[1-\sum \sigma_{x i}^{2} / \sigma^{\prime}{ }^{2}\right] \text {, where }
$$

$\mathrm{y}=\mathrm{x}_{1}+\mathrm{x}_{2}+\ldots \ldots \ldots \ldots+\mathrm{x}_{\mathrm{k}} ; \mathrm{k}=$ Number of items $(=20)$,

$\mathrm{x}_{\mathrm{i}}=$ the score of $\mathrm{i}$-th item,

$\mathrm{O}_{\mathrm{xi}}{ }^{2}=$ the variance of scores of i-th item,

$\mathrm{o}_{\mathrm{y}}^{2=}$ variance of $\mathrm{y}$.

Reliability had also been measured by Spearman-Brown formula, where splitting of tests were done by iterative methods proposed by Chakrabarty SN [2]. The formula for reliability coefficient is $\mathrm{R}=$, where $\mathrm{r}$ is the correlation coefficient of test of results of $1^{\text {st }}$ and $2^{\text {nd }}$ half.

\section{Results and Discussion}

The descriptive statistics of $x_{i}$ for different items were shown in Table1. Item1[Smoking cigarettes can cause health disorders], 2[The smoking persons possibly gets cough and rhinorrhea] and 7 [The number of inhaled cigarettes in a day is a factor that influences lung cancer incidence.] were evaluated more homogeneously compared to other items as the variances in the measurement of these 3 items were lowest compared to the variances of measurement of other items. Item 18 [Smokers can quit smoking easily whenever they wish] was measured more heterogeneously as the variance of measurement of this item was 0.808 . The variance of $y$ values was $\alpha_{y}^{2}=91.522$ and hence the Cronbach's alpha was found as $\alpha=0.94$ indicating that the evaluation of knowledge about health hazard of tobacco smoking was excellently reliable.

Table1: Descriptive statistics of $x_{i}$.

\begin{tabular}{|c|c|c|c|c|c|c|}
\hline Area of Evaluation & Item & Minimum & Maximum & Mean & Std. Deviation & $\alpha_{x i}{ }^{2}$ \\
\hline & 1 & 1 & 3 & 2.9207 & .35257 & .124 \\
\hline & 2 & 1 & 3 & 2.8623 & .3874 & .15 \\
\hline & 3 & 1 & 3 & 2.6821 & .54708 & .299 \\
\hline & 4 & 1 & 3 & 2.1481 & .75394 & .568 \\
\hline & 5 & 1 & 3 & 2.801 & .50784 & .258 \\
\hline & 6 & 1 & 3 & 2.712 & .54261 & .294 \\
\hline & 7 & 1 & 3 & 2.7135 & .56052 & .314 \\
\hline
\end{tabular}




\begin{tabular}{|c|c|c|c|c|c|c|}
\hline \multirow{13}{*}{$\begin{array}{l}\text { Knowledge of health hazard of } \\
\text { tobacco smoking }\end{array}$} & 8 & 1 & 3 & 2.8026 & .48111 & .231 \\
\hline & 9 & 1 & 3 & 2.7474 & .58198 & .339 \\
\hline & 10 & 1 & 3 & 2.5 & .75286 & .567 \\
\hline & 11 & 1 & 3 & 2.4482 & .72291 & .523 \\
\hline & 12 & 1 & 3 & 2.5751 & .68771 & .473 \\
\hline & 13 & 1 & 3 & 2.5736 & .7241 & .524 \\
\hline & 14 & 1 & 3 & 2.5335 & .74064 & .549 \\
\hline & 15 & 1 & 3 & 2.4755 & .79213 & .627 \\
\hline & 16 & 1 & 3 & 2.5226 & .78593 & .618 \\
\hline & 17 & 1 & 3 & 2.451 & .83792 & .702 \\
\hline & 18 & 1 & 3 & 2.1658 & .89875 & .808 \\
\hline & 19 & 1 & 3 & 2.2895 & .8804 & .775 \\
\hline & 20 & 1 & 3 & 2.283 & .88971 & .792 \\
\hline
\end{tabular}

The correlation coefficient of test results of first and second half was found out $\mathrm{r}=0.85$ and hence the reliability coefficient $\mathrm{R}=0.92$.

\section{Conclusion}

The assessment of knowledge about health hazard of smoking was evaluated by measuring 20 items. This type of assessment was needed to observe whether the higher level of awareness could lead the lower participation in smoking. But most of the students were at least medium level of awareness. No single case was identified as unaware of the problem. But a good number of students (32.9\%) were found smokers. Therefore, natural question was arisen whether students' awareness was evaluated reliably [16].

Awareness was evaluated using 20 items. Some of the items were evaluated more homogeneously by the students indicating that they agree highly in favor of danger of health hazard of tobacco smoking answering some of the items, specially item number 1, 2 and 7 , the responses of which were in favor of awareness of the problem. The overall assessment of awareness was excellent. From the study, it could be concluded that the students could be advised to avoid smoking as they were very much aware of the health hazard of tobacco smoking but not quitting the habit of smoking. As the students understood the problem, they could be motivated to get rid of the health hazard of tobacco smoking. However, their measure of awareness was reliable and there was possibility to get rid of the curse of disease originated from smoking. To avoid the problem of smoking, motivation campaign can be conducted to reduce tobacco consumption by informing the youth about the health hazard of smoking.

\section{Acknowledgement}

None

\section{Conflict of Interest}

Authors declare no conflict of interest.

\section{References}

1. Cronbach LJ (1951) Coefficient alpha and the internal structure of tests. Psychometrika 16(3): 297-334.

2. Chakrabarty SN (2013) Best split-half and maximum reliability. Jour Res \& Method in Education 3(1): 1-8.
3. Weir JP (2005) Quantifying test-retest reliability using the intraclass correlation coefficient and the SEM. Jour Strength Cond Res 19(1): 231 240 .

4. Safrit MJE (1975) Reliability Theory American Alliance for Health. Physical Education and Psychology.

5. Kaplan RM, Saccozzo DP (2001) Psychological testing Principle Application and Issues. ( $5^{\text {th }}$ edn). Wardworth, Belmont, USA.

6. Cochran WG (1941) The distribution of the largest of a set of estimated variances as a fraction of their total. Annals of Human Genetics 11(1): 47-52.

7. Cohen J (1960) A coefficient of agreement for nominal scales. Educational and Psychological Measurement 20(1): 37-46.

8. Cronbach LJ (1971) Test validation. In: Thorndike RL (Ed), Educational Measurement ( $2^{\text {nd }}$ edn). American Council of Education, Washington DC, USA

9. Kudar GF, Richardon MW (1937) The theory of the estimation of test reliability. Psychometrika 2(3): 151-160.

10.Guttman L (1945) A basis for analysis test-retest reliability. Psychometrika 10(4): 255-282.

11. Kelly MB (1977) A review of the observational data collection and reliability procedures reported in the journal of Applied Behavior Analysis. J Appl Behav Anal 10(1): 97-101.

12. Olga Towstopiat (1984) A review of reliability procedures for measuring observational agreement. Contemporary Educational Psychology 9(4): 333-352.

13. Maxwell AE, Pilliner AE (1968) Deriving coefficients of reliability and agreement for ratings. Br J Math Stat Psychol 21(1): 105-116.

14. Vacha-Hasse, T (1998) Reliability generalization: Exploring variance is measured error affecting score reliability across studies. Educational and Psychological Measurement 58(1): 6-20.

15. Webb NM, Shavelson RJ, Haertel EH (2006) Reliability Coefficients and Generalization Theory. Hand Book of Statistics.

16. Khatun M, Bhuyan KC (2014) Awareness of health hazard of tobacco consumption among students of American International University, Bangladesh, 13(1): 85-92

17. Mahato P (2012) Knowledge of Health Effect of Tobacco Smoking Among the Higher Secondary School Students. MPH Thesis, American International University, Bangladesh, Dhaka.

18. Bhuyan KC, Khatun M, Fardus J (2016) Awareness of health hazard of tobacco consumption among students of universities: A meta-analysis approach. Jour Stats Studies 33: 1-17.

19. Bhuyan KC, Urmi F (2018) Canonical correlation analysis to study the impacts of different social factors on awareness of health hazard of tobacco smoking and smoking habit. Biomed J Sci Tech Res 10(5): 1-5. 